\title{
Linx
}

Revue des linguistes de l'université Paris X Nanterre

$57 \mid 2007$

Études de syntaxe : français parlé, français hors de

France, créoles

\section{La variation en syntaxe dans le cadre de l'enseignement de la langue en Martinique}

Syntactic variation in the teaching of French in Martinique

\section{Jean-David Bellonie}

\section{OpenEdition}

\section{Journals}

Édition électronique

URL : http://journals.openedition.org/linx/288

DOI : $10.4000 /$ linx.288

ISSN : 2118-9692

Éditeur

Presses universitaires de Paris Nanterre

Édition imprimée

Date de publication : 1 décembre 2007

Pagination : 133-143

ISSN : 0246-8743

Référence électronique

Jean-David Bellonie, « La variation en syntaxe dans le cadre de l'enseignement de la langue en

Martinique », Linx [En ligne], 57 | 2007, mis en ligne le 15 février 2011, consulté le 15 mai 2020. URL

http://journals.openedition.org/linx/288 ; DOI : https://doi.org/10.4000/linx.288 


\title{
La variation en syntaxe dans le cadre de l'enseignement de la langue en Martinique
}

\author{
Jean-David Bellonie \\ Université Paris Onest Nanterre la Défense et MoDyCo \\ et Université Freibourg (Allemagne)
}

Les pratiques langagières en Martinique montrent des phénomènes de variation syntaxique spécifiques : la manifestation du contact de différentes formes de français, dont le français standard véhiculé notamment par l'école, et de différentes formes du créole, dont le créole standard également véhiculé surtout par l'école dans le cadre des cours de Langue et Culture Régionale - créole. Il en résulte des formes intermédiaires, interlectes, dont l'actualisation est en lien avec l'appréhension que les locuteurs ont des contraintes situationnelles.

Dans cet article, nous tenterons d'illustrer cette variation en nous intéressant aux prépositions, afin d'éclairer les différents sens que certaines d'entre elles peuvent prendre selon les situations. Ainsi, un locuteur n'interprètera pas une préposition de la même manière selon qu'il tente d'actualiser une forme proche du français standard, du créole standard ou une forme interlectale.

L'intérêt de telles observations réside notamment dans la prise en compte de l'enseignement du «français langue maternelle» en Martinique. Compte tenu des objectifs d'enseignement de la langue à l'école, la multiplicité des formes de créoles et de français en usage ne facilite pas la tâche des enseignants. Pourtant, la prise en compte de la variation et de ses effets pourrait permettre une approche plus souple, ce que nous tenterons de montrer à partir d'exemples de séquences en classe. 


\section{Les prépositions en français martiniquais (FM) et en français standard (FS)}

Les prépositions appartiennent à une catégorie de mots en français subissant une grande variation aussi bien en France continentale que dans d'autres aires de la francophonie (Gadet/Jones, 2008) ${ }^{1}$. Nous limiterons notre analyse à une comparaison entre la forme standard attendue et les formes qui s'actualisent en Martinique. Ceci étant posé, en contexte martiniquais, force est de constater que le type de relation induit par les prépositions (Prép), connecteurs et articulateurs n'est pas forcément le même selon qu'il s'agit de la forme standard attendue à l'école ou de la forme de français « local» ou « régional» qui s'actualise à l'oral, voire à l'écrit, en Martinique. Romani (2000 : 257 sq.) rappelle qu'en FM il y a un emploi régional des Prép dû à l'absence en créole martiniquais de à et de, marqueurs grammaticaux atones et désémantisés qui n’ont pas été conservés en créole. Celui-ci reconstruit un système sans de ni à (par ex. : man kay anvil/Fòdfwans = « je vais à Fort-de-France »). En FS, ces Prép dites « vides » ou « incolores » marquent une simple dépendance orientée entre deux constituants (par ex. : le directeur de l'usine/l'usine du directeur, Riegel et al, 2001 : 372). En FS, elles permettent l'évocation d'un mode de relation particulier entre les éléments, fonction qui explique que ces deux Prép soient parmi les mots français les plus utilisés (cf. Frantext ${ }^{2}$ ). On voit alors les problèmes posés par leur variation en FM et l'impact sur la maitrise du standard dans le cadre scolaire. En FM, il y a modification de la distribution des instructions sémantiques nécessaires au décodage de l'énoncé, et redistribution par rapport aux instructions de repérage des lieux (prépositions locatives et directionnelles) et de la destination de la personne (datif). On constate ainsi une confusion fréquente entre sur et sous; quant à dans, il cumule un emploi particulier pour l'indication du lieu d'où l'on vient (cf. Tableau 2), parallèlement aux emplois qu'on retrouve en FS.

Beaucoup de constructions FM peuvent être analysées comme des calques du créole, mais le système interlectal qui s'est mis en place en Martinique laisse supposer d'autres fonctionnements à l'œuvre, raison pour laquelle il faut les interpréter en fonction des instructions sémantiques (actancielles et spatiales) des verbes. Ainsi en est-il de cet exemple proposé par un de nos informateurs lycéen, qui constitue selon lui un prototype de non-maitrise du FS par un locuteur ayant le créole pour L1 :

(1) mets ton casque DANS $S^{3}$ ta tête

Bien que cet énoncé respecte la syntaxe du FS, il est jugé comme étant un «créolisme» inacceptable. Nous allons d'abord le regarder du point de vue du FS

\footnotetext{
${ }^{1}$ Il existe par ex. une extension d'utilisation de la préposition dans pour marquer la localisation aussi bien dans les créoles de l'Océan indien et de la zone caraỉbe, que dans le français de l'ouest de la France (Anjou et région de Nantes, cf. Chaudenson et al., 1993 : 102), ainsi qu'en français d'Amérique (en michif, selon Bakker, 1997).

${ }^{2}$ Les instructions officielles actuelles préconisent une liste de fréquence des mots à l'écrit (document d'application des programmes de l'école primaire française, 8 à 11 ans, cycle 3 ; 2004), collectée par E. Brunet sur Frantext (http://eduscol.education.fr/D0102/liste-motsfrequents.htm).

${ }^{3}$ Nous reproduisons la typographie de l'enquêté, avec les majuscules.
} 
avant de nous intéresser à son actualisation à l'oral et à l'écrit en contexte martiniquais. Selon les règles du FS, on note d'abord que le verbe mettre est trivalent. Il admet deux compléments (SN0 mettre SN1 Prép SN2). L'association de mettre + un casque + dans $S N$ (où $\mathrm{SN}=$ partie du corps) est rare en FS (une seule occurrence 4 sur 237.582.579 mots des textes du $20^{\text {ème }}$ siècle dans Frantext). (1) diverge du FS du point de vue syntaxique et sémantique en raison de contraintes non respectées imposées par le choix de la préposition locative dans permettant l'expression du rapport logique de « contenant/contenu » (C/c). Vandeloise (2006 : 252) rappelle qu'en français, le « site » de la préposition dans doit être placé après la préposition et qu'il représente le contenant. Ce dernier va déterminer la position du contenu, en position sujet. Mais cette règle ne permet pas d'expliquer la différence entre (2) et (3) :

\section{(2) L'ampoule est dans la douille}

(3) * La bouteille est dans le capuchon

(3) n'est pas acceptable, alors que du point de vue de la représentation spatiale, les relations ampoule/douille et bouteille/capuchon sont semblables. Cela relève d'un autre niveau, celui de la différence de relation dynamique. En effet, en (2) c'est la position de la douille qui détermine celle de l'ampoule, tandis qu'en (3) c'est la position de la bouteille qui est déterminante pour le capuchon. D'où une définition de dans en termes de relation contenant/contenu tenant compte de l'aspect dynamique d'une force exercée, en plus de la relation d'inclusion qui concerne les usages concrets de la préposition. L'interaction dynamique est un trait dominant de la relation $\mathrm{C} / \mathrm{c}$, le contenant contrôlant les mouvements du contenu. C'est pourquoi (3) est peu acceptable en FS, et cela explique aussi pourquoi (1) est perçu comme étrange : la tête joue le rôle d'un site qui détermine la position de la cible, le casque, qui exerce une force sur la tête et détermine sa position. On devrait donc avoir :

\section{(1') mets ta tête dans ton casque}

Cependant, mettre construit plutôt son SN2 avec sur (TLFi5 $\left.{ }^{5}\right)$ pour indiquer le contact avec la partie du corps. Ainsi, on aura plus souvent un énoncé du type :

\section{(1") mets ton casque (sur ta tête)}

où sur ta tête est facultatif (mets/enfile ton casque avec ellipse du syntagme prépositionnel, l'élément non exprimé étant récupérable à partir du contexte situationnel ${ }^{6}$ ou linguistique). En Martinique, cet énoncé est un message publicitaire diffusé à la télévision pour sensibiliser les motards au port du casque. (1) ne peut pas être interprété comme un calque d'une structure syntaxique du créole, où on aurait mété kas-ou (kas-la) anlè (asi) tèt-ou ${ }^{7}$, avec les prépositions anlè ou asi qui correspondraient en

\footnotetext{
4 «Cyrano, le décoiffant et lui mettant son casque dans la main. » Extrait de l'acte IV, scène 3 de Cyrano de Bergerac d'Edmond Rostand (1898 : 159). Voir cependant note 1.

5 Trésor Linguistique de la langue Française informatisé.

${ }^{6}$ Pour être efficace, le casque doit se porter sur la tête, et non au bras du conducteur par ex.

${ }^{7}$ Énoncés proposés par des informateurs créolophones adultes à qui nous avions posé la question de façon informelle.
} 
FS à sur. Pour autant, comme en français, l'option la plus probable serait l'économie du complément locatif : mété kas-ou («mets ton casque ») ou mété kas-la ${ }^{8}$ (" il faut mettre le casque ") comme on peut le lire sur des panneaux publicitaires de prévention routière : OU JA SAV : MÉTÉ $K A S^{\prime} L A \grave{A}$ (« tu sais déjà ce qu'il faut faire : mettre son casque $»)$.

Cette analyse va dans le sens de l'hypothèse de Romani (2000). Nous allons illustrer nos propos à partir de l'analyse sémantique des relations locatives que l'on trouve dans nos corpus oraux constitués d'enregistrements dans des classes, où il était demandé aux enfants de raconter une histoire à partir d'images sans texte. Les contraintes de genre discursif de l'exercice imposaient aux informateurs un repérage spatio-temporel.

\section{Analyse sémantique des relations locatives}

De façon générale, les analyses sémantiques des relations locatives cherchent à isoler les contraintes devant être satisfaites par une configuration spatiale constituée par : une entité à localiser (« entité-cible ») ; une entité de référence (« entité-site ») avec la mention des différentes propriétés des entités spatiales en présence. Par exemple, les emplois localisateurs de à permettent de prendre en compte les critères de lieu $(+\mathrm{ou}-$ fixes), de portion d'espace ( + ou - spécifiée) et d'objet (non fixité et absence de portion d'espace). La préposition dans permet d'isoler les notions de substance, de portion de matière et de collection. D'autres critères pourraient être pris en compte selon les propriétés sémantiques associées aux configurations spatiales décrites, ce qui amène Aurnague et al. (2005) à postuler que : « [...] la classification des entités sous-tendant les descriptions spatiales ne doit pas être conçue comme quelque chose de rigide/ immuable (et extérieur au système linguistique) mais comme un "point de vue » adopté par la langue au moment d'identifier une entité (point de vue qui est, bien sûr, conditionné par les propriètés du référent considéré). »

De façon simplifiée, on peut considérer avec Romani (2000: 259 sq.) que trois catégories d'instructions sémantiques sont nécessaires à la traduction des rapports de direction et de localisation dans l'espace (encodage et décodage) : du point de vue actanciel, une instruction S1 [+ déplacement] ou [- déplacement] représentée par les verbes aller vs être, avec une opposition des cas directionnels et locatifs; du point de vue spatial, l'instruction S2 "position » qui permet d'exprimer un rapport entre un procès et un lieu, et va configurer ce dernier comme étant " clos/ouvert » ou «haut/bas » par ex. ; la troisième instruction $\mathrm{S} 3$ est corrélée à $\mathrm{S} 1$ " déplacement ». Dans ce cas de figure, plusieurs lieux successifs sont mis en scène et $\mathrm{S} 3$ permet de spécifier lequel est actualisé : S3 correspond à l'« aspect » en tant que marqueur du caractère initial, intermédiaire ou final du lieu désigné par rapport au procès exprimé. L'auteur prend l'exemple du syntagme FS sortir de où l'on peut isoler deux types d'instructions portées par sortir : S1 « aller » et S2 « hors d'un lieu clos » (voir la définition du Petit Larousse : " quitter un lieu pour aller dehors »). La préposition de exprime l'aspect « origine ».

${ }^{8}$ Avec le même phénomène d'ellipse du syntagme qu'en français. 
Tableau 1:

Instructions sémantiques portées par les prépositions (d'après Romani, 2000)

\begin{tabular}{|l|l|l|}
\hline $\begin{array}{c}\text { S1 } \\
\text { déplacement }\end{array}$ & \multicolumn{1}{|c|}{$\begin{array}{c}\text { S2 } \\
\text { position / configuration }\end{array}$} & \multicolumn{1}{c|}{$\begin{array}{c}\text { S3 } \\
\text { aspect }\end{array}$} \\
\hline$[-]$ être & dans, sur, sous, à, ... & - \\
\hline$[+]$ aller & $\grave{a}$, dans, sur, de...à, à...par \\
\hline$[+]$ sortir $[$ clos -$]$ extérieur $]$ & de, en, par, ... \\
\hline$[+]$ descendre [haut - $]$ bas $]$ & de, à, par, ... \\
\hline
\end{tabular}

Nous nous proposons d'illustrer cette analyse à partir d'un relevé d'énoncés en FM comportant l'emploi de prépositions locatives/directionnelles dans 20 récits d'enfants en français. Pour l'analyse de la référence spatiale, limitée ici au simple repérage des prédicats et à leur configuration, nous nous sommes appuyés sur des travaux en acquisition (Hickmann / Hendriks / Roland, 1998). Nous avons analysé les récits de façon à repérer tout d'abord les situations contenant un prédicat statique ou dynamique. Par "prédicat ", il faut entendre la totalité du syntagme verbal incluant la racine verbale et les «satellites » (Prép et particules). Deux types d'entités étaient repérés :

- le site : entité mise en mot et/ou localisée par le prédicat ;

- le référent : entité à laquelle la cible est reliée par différents types de relation.

Trois configurations ont alors été distinguées :

- la localisation statique (être sur le lit);

- le mouvement à l'intérieur d'une localisation (courir dans la cuisine);

- le mouvement impliquant un changement de localisation (il est entré dans la cuisine en courant).

Dans les deux tableaux qui suivent, le tableau (3), illustratif et comparatif, propose d'autres exemples d'actualisation de la variation syntaxique de dans issus d'enregistrements de locuteurs martiniquais avec d'autres paramètres situationnels (environnement discursif, contexte et contraintes de production variés). Quant au tableau (2), il regroupe les emplois divergents de dans en fonction du prédicat dans le corpus de récits. Dans la première colonne sont présentés les prédicats avec dans. Nous précisons le nombre d'occurrences du prédicat selon la configuration définie et le nombre d'attestations dans les récits des élèves. Puis sont donnés quelques éléments de définition tirés du TLFi, mentionnant parfois la Prép attendue en FS avec ce type de prédicat. Les trois colonnes suivantes regroupent des exemples d'occurrences attestées dans le corpus (élèves) classées selon trois types de configuration : statique, dynamique et (+/-) changement.

Tableau 2 Emplois divergents de dans dans le corpus de récits d'élèves

\begin{tabular}{|l|l|l|l|}
\hline \multicolumn{1}{|c|}{ Prédicat + Prép dans } & statique & $\begin{array}{c}{[+] \text { dynamique }} \\
{[-] \text { changement }}\end{array}$ & $\begin{array}{c}{[+] \text { dynamique }} \\
{[+] \text { changement }}\end{array}$ \\
\hline $\begin{array}{l}\text { Sortir dans }(\text { sur } 49 \text { occurrences, } 5 \text { en } \mathrm{FM}) \\
\text { lieu d'origine de }(\mathrm{S} 1 \text { « clos - extérieur })\end{array}$ & & & $\begin{array}{l}\left(\mathrm{E} 1{ }^{9}\right) \text { un animal qui } \\
\text { sortait dans le terrier }\end{array}$ \\
\hline $\begin{array}{l}\text { Emmener dans }(\text { sur } 4 \text { occurrences, } 2 \text { en } \\
\mathrm{FM}) \text { localisation / destination }(\text { sur }),\end{array}$ & & $\begin{array}{l}\text { (E2) la bête l'emmena } \\
\text { dans un rivage }\end{array}$ \\
\hline
\end{tabular}

${ }^{9}$ Les élèves sont identifiés par l'initiale « E » suivie d’un numéro (1 à 9). 


\begin{tabular}{|c|c|c|}
\hline $\begin{array}{l}\text { mener avec soi d'un endroit dans un } \\
\text { autre }\end{array}$ & & \\
\hline $\begin{array}{l}\text { Se relever dans (sur } 1 \text { occurrence, } 1 \text { en } \mathrm{FM} \text { ) } \\
\text { remettre debout quelqu'un ou quelque } \\
\text { chose qui est tombé }\end{array}$ & & $\begin{array}{l}\text { (E3) le garcon se relève } \\
\text { dans l'eau }\end{array}$ \\
\hline $\begin{array}{l}\text { Aller (+ V-inf) dans (sur } 63 \text { occ., } 1 \text { en } \\
\text { FM) }\end{array}$ & & $\begin{array}{l}\text { (E4) il est allé regarder } \\
\text { dans la fenêtre avec son } \\
\text { chien }\end{array}$ \\
\hline $\begin{array}{l}\text { Monter dans (sur } 39 \text { occurrences, } 2 \text { en } \\
\text { FM) sur, localisation ( } 1 \text { « haut - bas ») se } \\
\text { déplacer dans un mouvement ascendant, } \\
\text { s'élever dans un espace sans limite } \\
\text { précise }\end{array}$ & & $\begin{array}{l}\text { (E5) il (montit) dans un } \\
\text { rocher énorme }\end{array}$ \\
\hline $\begin{array}{l}\text { Glisser dans (sur } 1 \text { occurrence, } 1 \text { en FM) } \\
\text { se déplacer sans secousse (sur la surface } \\
\text { d'un corps lisse ou le long d'un autre } \\
\text { corps) }\end{array}$ & & $\begin{array}{l}\text { (E6) le garcon a glissé } \\
\text { dans la tête du [sErs] }\end{array}$ \\
\hline $\begin{array}{l}\text { Rester dans (sur } 3 \text { occurrences, } 1 \text { en FM) } \\
\text { continuer d'être de façon }+ \text { ou }- \\
\text { prolongée ou durable, dans un lieu ou } \\
\text { dans un état }\end{array}$ & $\begin{array}{l}\text { (E7) il est resté } \\
\text { dans la vitre }\end{array}$ & \\
\hline $\begin{array}{l}\text { Avoir dans (sur } 41 \text { occurrences dont } 37 \text { il } \\
\text { y a/avait, } 2 \text { en FM) }\end{array}$ & $\begin{array}{l}\text { (E8) le chien avait } \\
\text { toujours le bocal } \\
\text { dans la tête }\end{array}$ & \\
\hline Etre dans (sur 63 occurrences, 1 en FM) & $\begin{array}{l}\text { (E9) le jeune } \\
\text { garçon est dans sur } \\
\text { sur sur sa tête }\end{array}$ & \\
\hline
\end{tabular}

Nous avons relevé dans le corpus neuf prédicats susceptibles d'être employés de façon non standard avec dans. Leurs configurations sont les suivantes :

- deux prédicats ayant les traits $(+)$ dynamique $(+)$ changement : sortir, emmener;

- quatre prédicats avec les traits $(+)$ dynamique $(-)$ changement : se relever, aller, monter, glisser;

- trois prédicats statiques : rester, avoir, être.

L'emploi divergent du FS de la Prép dans est stigmatisé par les enseignants, alors qu'il semble parfaitement fonctionnel et ce, dans de nombreuses situations de communication, comme le montrent les exemples du tableau ci-après. L'attitude normative des enseignants semble d'autant plus problématique qu'eux-mêmes sont susceptibles d'actualiser cette variante sans en avoir conscience (énoncés 3 et 4). C'est l'effet de « double-bind» (injonctions paradoxales) que l'on peut résumer en « fais ce que je dis et pas ce que je fais ». La variante syntaxique avec dans est susceptible d'apparaittre dans les environnements syntaxiques variés présentant des configurations sémantiques particulières. Les situations de communication où elle s'actualise peuvent relever de l'immédiat comme de la distance communicative (Koch/Esterreicher, 2001), distance qui s'illustre dans les enregistrements d'élèves : un récit oral en contexte scolaire à partir d'un support face à un interlocuteur-enquêteur adulte en position d'évaluer la production orale.

Les enseignants informés et sensibilisés à la variation et au fonctionnement particulier de dans seront plus à même de traiter différemment ce type de phénomène, perçu dans le cadre scolaire comme une erreur, constitutif d'un instant pidgin (Le Page, 1979) contraint par une situation de production précise : le destinataire du message doit partager les règles d'utilisation des variantes interlectales que l'on trouve en 
situation de créolophonie ; la réalisation de cette variante apparait comme un possible communicationnel parmi ceux offerts par le large système de variation interlectal. L'erreur réside dans son inadaptation aux attentes de l'institution. Cependant, le nombre d'occurrences dans le corpus de récits d'enfants reste limité (15), ce qui prouve que ces élèves de milieu modeste maitrisent également la variété standard dans des situations contraintes par des paramètres précis. Il aurait fallu pouvoir contraster ces résultats avec de tels récits à l'écrit. La comparaison avec les constructions telles qu'elles apparaissent dans d'autres situations de communication, produites par des locuteurs soumis à d'autres types de contraintes, est intéressante parce qu'elle permet une mise en relation des formes et des contextes de production.

Tableau 3 Exemples d'emplois divergents de dans attestés ${ }^{10}$ dans nos autres corpus

\begin{tabular}{|c|c|c|c|c|c|c|c|}
\hline \multirow{2}{*}{$\begin{array}{c}\begin{array}{c}\text { Situation } \\
\text { de }\end{array} \\
\text { communi- } \\
\text { cation / canal } \\
\end{array}$} & \multicolumn{5}{|c|}{ Exemples divergents du FS } & \multirow{2}{*}{$\begin{array}{l}\text { Exemples en } \\
\text { créole }\end{array}$} & \multirow{2}{*}{$\begin{array}{l}\text { Gloses en } \\
\text { français }\end{array}$} \\
\hline & & $\begin{array}{c}\text { SN1 } \\
\varnothing\end{array}$ & $\mathrm{V}$ & $\begin{array}{c}\mathrm{SN} 2 \\
\mathrm{SN} 2+\mathrm{SP} \\
\mathrm{SP} \varnothing \\
\end{array}$ & $\begin{array}{l}\text { Prép }+ \\
\text { SN3 }\end{array}$ & & \\
\hline $\begin{array}{l}\text { Commentaire } \\
\text { métalinguisti } \\
\text { que / écrit } \\
\text { (questionnai } \\
\text { re rempli en } \\
\text { classe) }\end{array}$ & $(\mathrm{I} 1)^{11}$ & $\varnothing$ & mets & ton casque & $\begin{array}{l}\text { dans ta } \\
\text { tête }\end{array}$ & $\begin{array}{l}\text { (I6) mété kas-ou } \\
\text { asi tèt-ou }\end{array}$ & $\begin{array}{l}\text { mets/enfile ton } \\
\text { casque (sur tête) }\end{array}$ \\
\hline $\begin{array}{l}\text { Récit / oral } \\
\text { (groupe, } \\
\text { chez } \\
\text { l'informatrice) }\end{array}$ & (I2) & $\begin{array}{l}\text { le } \\
\text { petit } \\
\text { gargon }\end{array}$ & reste pris & $\varnothing$ & $\begin{array}{l}\text { dans les } \\
\text { cornes }\end{array}$ & $\begin{array}{l}\text { (I2) mi i pri adan } \\
\text { an serf adan kon } \\
\text { an serf }\end{array}$ & $\begin{array}{l}\text { le petit garçon } \\
\text { reste coincé } \\
\text { entre les cornes }\end{array}$ \\
\hline $\begin{array}{l}\text { Discours } \\
\text { didactique / } \\
\text { Oral } \\
\text { (groupe, en } \\
\text { classe) }\end{array}$ & (I3) & tu & n'as pas & $c ̧ a$ & $\begin{array}{l}\text { dans ta } \\
\text { mémoire? }\end{array}$ & $\begin{array}{l}\text { ? ou pa ni sa } \\
\text { an/adan tèt-ou } \\
?\end{array}$ & $\begin{array}{l}\text { tu n'as pas ça en } \\
\text { mémoire/en tête } \\
\text { ? }\end{array}$ \\
\hline $\begin{array}{l}\text { Biographie } \\
\text { langagière / } \\
\text { oral (tête à } \\
\text { tête, chez } \\
\text { l'informateur) }\end{array}$ & (I4) & si elle & $\begin{array}{l}\text { venait } \\
\text { trouver }\end{array}$ & quelqu'un & $\begin{array}{l}\text { dans sa } \\
\text { place }\end{array}$ & $\begin{array}{l}\text { ? si i té ka } \\
\text { touvé/wè an } \\
\text { moun an plas li }\end{array}$ & $\begin{array}{l}\text { si elle découvrait } \\
\text { quelqu'un à sa } \\
\text { place }\end{array}$ \\
\hline $\begin{array}{l}\text { Interview / } \\
\text { oral (tête à } \\
\text { tête, chez } \\
\text { l'informateur) }\end{array}$ & (I5) & tu & vas voir & $\begin{array}{l}\text { des gens } \\
\text { avec des } \\
\text { sébago }\end{array}$ & $\begin{array}{l}\text { dans } \\
\text { leurs } \\
\text { pieds }\end{array}$ & $\begin{array}{l}\text { ou ké wè moun } \\
\text { (kea pòté/épi) } \\
\text { sébago an/adan } \\
\text { pié yo }{ }^{12}\end{array}$ & $\begin{array}{l}{[\ldots] \text { avec des }} \\
\text { Sébago aux } \\
\text { pieds }\end{array}$ \\
\hline
\end{tabular}

Tenir compte de la variation sémantique des prépositions prend tout son sens dans l'enseignement dans une région comme la Martinique. Certes, on y enseigne un français standard, mais le contact avec le créole et l'émergence d'interlectes caractérisés

\footnotetext{
${ }^{10}$ Les exemples attestés sont en italiques. Le point d'interrogation en début d'énoncé indique que l'exemple est construit.

${ }^{11}$ Les informateurs sont identifiés par l'initiale «I $~$ » suivie d'un chiffre.

12 Cf. Cervinka (1996 : $122 ; 125)$.
} 
notamment par des emplois particuliers des prépositions, exigent d'adapter le discours pédagogique. Or, bien souvent, l'idéologie du standard imprègne les pratiques des enseignants au point où, même pour des enseignants de Langue et Culture Régionale sensibilisés au contact des langues et à la variation, l'usage d'une unité de la langue reconnue comme acceptable en FS à des fins communicatives autres que celles attendues se trouve invalidé.

En guise de conclusion, nous nous proposons d'observer comment les enseignants que nous avons rencontrés traitent ce qui est perçu comme une erreur de l'élève, là où manifestement il est question d'effets du contact des langues. Les exemples ne traitent pas spécifiquement de la préposition, mais on comprendra que les emplois divergeants des prépositions connaissent le même sort.

\section{Intégrer la variation à l'enseignement}

Étant donné que l'objet de l'enseignement de la langue à l'école en Martinique comme en métropole vise le FS, tout écart est perçu comme une erreur. Si on ne parle pas de «faute », c'est qu'on entend comprendre les chemins qu'empruntent les élèves lorsqu'ils proposent des formes différentes. En l'occurrence, il est souvent question d'une mauvaise appréhension des contraintes réelles ou fabriquées qui donnent lieu à l'actualisation de la forme standard. Partant, lorsque les élèves martiniquais proposent des constructions syntaxiques propres au FM, une remédiation pertinente devrait s'envisager en s'interrogeant sur la façon de traiter ce qui apparait comme des erreurs. Le traitement de l'erreur et notamment des interférences est considéré comme révélateur des conceptions didactiques, linguistiques et du rapport à la norme des enseignants en même temps que les reprises qu'ils font ou non témoignent de décisions interactives. Plus un enseignant est expérimenté et plus il connaît sa classe, plus il est à même de gérer les interactions et les erreurs dans l'activité en fonction des objectifs de celle-ci.

Nos exemples A à $\mathrm{D}^{13}$ proviennent d'enregistrements effectués dans une classe par un enseignant $(\mathrm{M})$ qui assure aussi des cours de créole. Ce choix est dicté par l'idée qu'un tel enseignant est potentiellement plus enclin à admettre la variation. Les situations de classe sélectionnées nous intéressent dans la mesure où l'enseignant est confronté à des logiques de fonctionnement ne lui permettant pas d'appréhender de façon optimale les erreurs de ses élèves (E). L'analyse des interactions de classe permet de noter plusieurs types de reprises et/ou corrections au cours de différentes séances. Celles-ci interviennent de façon systématique et oscillent entre souplesse et rigidité, avec une difficulté dans la présentation de la variation aux élèves. On trouve en particulier une rectification des «scories » de l'oral, rarement explicitée, récurrente dans tous les enregistrements :

\footnotetext{
13 Conventions de transcription : «+» pour les pauses de moins d'une seconde et indications entre parenthèses pour les pauses plus longues, par ex. «(1.3s)»; «? » pour une intonation montante (question) ; « = » pour un enchainement rapide ; «X » pour les syllabes incompréhensibles ; usage des majuscules pour noter les phénomènes de saillance perceptuelle (ex. : chez ta TANte) ; «::» pour les allongements; parenthèses simples pour les segments incertains et doubles pour les commentaires divers ; « $[\ldots]$ » pour les chevauchements ; «EE » pour le groupe d'élèves.
} 
- au niveau locutoire et prosodique (« euh »), accent chantant des élèves, interférence avec la phonologie du créole ;

- des rectifications lexicales ou morpho-syntaxiques parfois dues aux interférences avec le créole : rappel implicite qu'il existe des registres de langue (tatie vs tante, maman / manman vs mère); rectification d'ordre morphosyntaxique: dans l'extrait $\mathrm{C}$, l'enseignant n'entend pas l'élève prononcer la Prép de devant migan (phénomène d'assimilation du $e$ muet entre les consonnes « $\mathrm{d} »$ et $« \mathrm{f} »)$.

(A)

\begin{tabular}{|l|l|}
\hline M & est-ce qu'il y a qui travaillent avec une voisine ? (1.3s) \\
\hline E10 & non \\
\hline M & un voisin ? \\
\hline E11 & non \\
\hline M & non +++ avec qui est-ce que tu as travaillé ? \\
\hline E13 & XX \\
\hline M & parle plus fort \\
\hline E13 & ma mère \\
\hline M & ta mère ++ mais lorsque tu montais hier tu allais où ? $(1 \mathrm{~s})$ \\
\hline E13 & chez ma tatie \\
\hline M & chez ta tatie où ? + chez ta TANte \\
\hline E13 & oui \\
\hline M & $\begin{array}{l}\text { pas ta }+ \text { mère }++ \text { tu n'es pas toute seule à travailler ? ++ donc tu as } \\
\text { travaillé avec ta tante ? }++ \text { pas avec ta mère }+++\end{array}$ \\
\hline
\end{tabular}

(B)

\begin{tabular}{|l|l|}
\hline M & BIEN +++ oui ? \\
\hline E6 & euh:: mm:: + \\
\hline M & pourquoi que tu commences par euh ? (1.1s) euh:: (2s) \\
\hline E6 & ma maman vo- a::vait oublié de me \\
\hline M & = laisse maman ++ mam- laisse manman ma MEre \\
\hline E6 & $\begin{array}{l}\text { d'accord monsieur + ma mère a oublié de:: d'écrire + de me donner:: } \\
\text { la recette du colombo et:: + elle a (oublié de) XX }\end{array}$ \\
\hline
\end{tabular}

(C)

\begin{tabular}{|l|l|}
\hline E14 & euh:: $\mathrm{mm}+\mathrm{j}$ 'ai apporté la recette sur + le migan de fruit à pain \\
\hline $\mathrm{M}$ & le migan DE (1.2s) répète le \\
\hline E14 & migan de fruit à pain \\
\hline
\end{tabular}


(D)

\begin{tabular}{|c|c|}
\hline M & tu as vu hier soir? \\
\hline E5 & oui:: \\
\hline $\mathrm{M}$ & c'était intéressant? \\
\hline E5 & oui [je suis allée ] \\
\hline $\mathrm{E} 2$ & [ monsieur ] \\
\hline $\mathrm{M}$ & $\begin{array}{l}\text { quand- }++ \text { quand:: au moment quand je s:: je savais pas je suis allée }+ \text { j'ai } \\
\text { regardé le début et puis:: + un:: bon moment après:: j'ai pris sommeil }\end{array}$ \\
\hline $\mathrm{M}$ & $\begin{array}{l}\text { j'ai PRIS sommeil ? quoi qu'est-ce que tu as avec sommeil ? + j'ai PRIS } \\
\text { sommeil ? + comment que tu- alors- }+ \text { j'ai [pris:: }\end{array}$ \\
\hline E8 & [je me suis ] endormi::) \\
\hline $\mathrm{M}$ & j'ai pris le dictionnaire +++ j'ai pris sommeil +++ \\
\hline $\mathrm{E} 8$ & ((en chuchotant)) je me suis endormi \\
\hline $\mathrm{M}$ & où que tu l'as pris ? (1.2s) \\
\hline E5 & je l'ai pris \\
\hline $\mathrm{M}$ & [ c'est des XX J'AI pris sommeil c'est quel verbe ] le verbe ? ++ \\
\hline E15 & [ monsieur je XXX \\
\hline E5 & [euh:: \\
\hline $\mathrm{E}$ & [ prendre un dictionnaire ] \\
\hline E5 & PREN[ dre \\
\hline M & [ PREN ]dre + alors comment que tu as pris $?++$ \\
\hline E5 & ((baissant la voix $)$ je s::ais pas ++ \\
\hline $\mathrm{M}$ & c'est toi qui a dit j'ai pris ++ \\
\hline E15 & je me [ S::UIS \\
\hline $\mathrm{M}$ & $\begin{array}{l}\text { [ PRENDS ] quelque chose pour moi essaie de prendre quelque [ chose } \\
\text { (ici) ] }\end{array}$ \\
\hline $\mathrm{E} 8$ & {$[\mathrm{XXX}$} \\
\hline $\mathrm{M}$ & $\begin{array}{l}=\text { tu as } \mathrm{p}^{-}+\mathrm{j}^{\prime} \text { ai pris le livre }++ \text { comment tu peux pris sommeil qu'est-ce } \\
\text { qu'on dit:: ? }\end{array}$ \\
\hline E15 & je me suis endo::r[ $\mathrm{mi}]$ \\
\hline $\mathrm{M}$ & [je ] me suis endormie [ c'est vrai c'est ça hein + ] \\
\hline $\mathrm{E} 8$ & [XXX \\
\hline $\mathrm{M}$ & $\begin{array}{l}\text { mais même avec des collègues hein }+ \text { tu sais j'ai pris sommeil sur le canapé:: } \\
++\end{array}$ \\
\hline E16 & OUI:: moi [ aussi (je dis, j’avais dit) ça:: ] \\
\hline $\mathrm{M}$ & $\begin{array}{l}\text { [hein le sommeil m'a ] pris sur le:: canapé:: ((en riant)) c'est } \\
\text { vrai }+ \text { alors évitons hein ces choses là d'accord ? ++ évitons }\end{array}$ \\
\hline
\end{tabular}

Ce qui pose problème dans cet extrait est l'attitude normative de l'enseignant qui, bien que tentant de donner une légitimité à la proposition de l'enfant, n'explique pas pourquoi il n'accepte pas prendre sommeil. C'est un autre exemple de « double bind », car de son propre aveu cette expression lui est familière dans ses pratiques quotidiennes. Il semblerait plus pertinent de faire réfléchir l'enfant au caractère 
interlectal de sa production et de son inadéquation aux attentes de l'école, c'est-à-dire ce qui sera évalué en cours de Langue et Culture Régionale - Créole (la forme standard du créole martiniquais) ou en cours de français (la forme standard du français). Ainsi, on peut demander à l'élève de dire la même chose en créole, en l'occurrence pwan somey. Ce qui confirme que prendre sommeil prend appui sur la forme créole. Dans un second temps, il est indispensable de ne pas invalider les mots français prendre et sommeil. Il faudra amener les enfants à aller chercher dans le dictionnaire les définitions de ces deux mots, afin qu'ils appréhendent par eux-mêmes l'impossibilité, en FS, de combiner prendre et sommeil. Rien n'empêche par la suite de réfléchir avec les élèves aux raisons pour lesquelles prendre sommeil peut faire sens même pour des noncréolophones en exploitant le caractère poétique de cette expression.

\section{RÉFÉRENCES BIBLIOGRAPHIQUES}

Aurnague, M. / Hickmann, M. / Vieu L. (2005) : «Les entités spatiales dans la langue : étude descriptive, formelle et expérimentale de la catégorisation », in Thinus-Blanc, C. / Bullier, J. (eds) : Agir dans l'espace, Editions de la Maison des Sciences de l'Homme, Paris : 217-232.

CervinkA, B. (1996) : "Regard sur quelques emplois de prépositions en créole martiniquais », in Véronique, D. (éd.) : Matériaux pour l'étude des classes grammaticales dans les langues créoles, P.U.P, Aix-en-Provence : 113-134.

BAKker, P. (1997) : A Language of Our Own: The Genesis of Michif, the Mixed Cree-French Language of the Canadian Metis, Oxford University Press, New-York.

Chaudenson, R. / Mougeon, R. / Beniak, E. (1993) : Vers une approche panlectale de la variation du français, Didier Erudition, Paris.

Gadet, F. / Jones, M. C. (2008) : «Variation, Contact and Convergence in French Spoken Outside France », Journal of language contact : 238-248.

Hickmann, M. / Hendriks, H. / Roland, F. (1998) : «Référence spatiale dans les récits d'enfants », Langue Française 118 : 104-126.

Koch, P. / CEsterreicher, W. (2001) : « Gesprochene und geschriebene Sprache / Langage parlé et langage écrit », in Holtus, G. / Metzeltin, M. / Schmitt, C. (éd.) : Lexikon der romanistischen Linguistik, vol. I.2, Max Niemeyer Verlag, Tübingen : 584-627.

LE PAGE, R. (1979) : « Reviews », Journal of Linguistics 15 : 168-169.

Riegel, M. / Pellat, J.-C. / Rioul, R. (2001) : Grammaire méthodique du français, Quadrige- PUF, Paris (réédition de 1994).

Romani, J.-P. (2000) : L’interlecte Martiniquais. Approches sociolinguistiques des rapports langue/ idéologie, Thèse de Doctorat, Université de Rouen.

VAndeloise, C. (2006) : De la distribution à la cognition, L'Harmattan, Paris. 
